\title{
Combinación de nuevas técnicas electrofisiológicas y de imagen en el estudio de la función de la corteza visual primaria
}

\author{
J. Mariño, J. Schummers, M. Sur
}

\author{
COMBINATION OF NEW ELECTROPHYSIOLOGICAL AND IMAGING TECHNIQUES \\ IN THE STUDY OF PRIMARY VISUAL CORTEX FUNCTION
}

\begin{abstract}
Summary. Introduction. This work summarizes current research focused on explaining orientation selectivity of primary visual cortex (V1), and describes the electrophysiological and imaging techniques than are being used. Development. The study of orientation selectivity in V1 is key to understanding the cortical mechanisms implicated in the processing of sensory information, but this enterprise has proved more challenging than previously thought and there is no consensus about the best model to explain V1 neurons' activity. Ongoing research is focused on determining the importance of the different inputs that a cortical cell receives (thalamic and lateral cortical inputs), and their link to cortical architecture. To achieve that, current research is combining optical imaging techniques with intracellular recordings of V1 neurons. Recent findings have found differences in the synaptic integration performed by neurons located in the iso-orientation domains vs orientation centers of the functional V1 map. Conclusions. Data describing synaptic activity combined with the cortical functional structure are yielding new clues about V1 computation, suggesting that there is more than one mechanism capable of generating orientation selectivity. [REV NEUROL 2003; 36: 944-50]
\end{abstract}

Key words. Optical imaging of intrinsic signals. Orientation map. Primary visual cortex. Synaptic effects. V1.

\section{INTRODUCCIÓN}

Aunque se conoce con bastante precisión la actividad de ciertas regiones corticales, todavía se está muy lejos de entender los mecanismos funcionales y estructurales subyacentes. Al contrario de lo que ocurre con el estudio de otros sistemas complejos, en donde el conocimiento de la estructura ha llevado de manera más o menos automática al descifrado de la función, en el estudio de la corteza cerebral se ha avanzado en la comprensión de ciertos aspectos básicos de la actividad celular cuando todavía existe un conocimiento limitado de la estructura que la origina, a pesar de la aparente organización estereotipada [1]. Este es el caso de las células de la corteza visual primaria (V1, la región más intensamente estudiada del cerebro de los mamíferos), cuya respuesta ante estímulos simples se conoce bastante bien y se puede encontrar en cualquier libro de texto desde los trabajos de Hubel y Wiesel en los años sesenta [2], sin embargo, se está empezando a descifrar la arquitectura y función de la microcircuitería que da lugar a esas respuestas y todavía faltan por demostrar las conexiones anatómicas responsables de propiedades básicas como la selectividad a la orientación, disparidad binocular, selectividad a la dirección, selectividad a la frecuencia espacial y adaptación al contraste, entre otras.

El estudio de las propiedades funcionales de V1 y, en concreto, los mecanismos corticales que producen la selectividad a la orientación, se considera un primer paso para conocer los procesos de computación e integración de la información sensorial llevados a

Recibido:04.04.03. Aceptadotras revisión externa sinmodificaciones: 04.04.03. Department Brain and Cognitive Sciences. Massachusetts Institute of Technology (MIT). Cambridge, MA, EEUU.

Correspondencia: Dr. Jorge Mariño. Dep. Brain and Cognitive Sciences, MIT. 45 Carleton St, E25-235. Cambridge MA, 02139 EEUU. Fax: 6172539 829. E-mail: xurxo@mit.edu.

Agradecimientos. A los compañeros del NEUROCOM, por sus críticas y sugerencias. El trabajo se ha desarrollado gracias a proyectos de investigación del NIH (EEUU), a una beca posdoctoral del MECD (JM) y una beca predoctoral del Howard Hughes Medical Institute (JS).

C) 2003, REVISTA DE NEUROLOGÍA cabo por la corteza $[3,4]$. Con ello se trata de conocer las estrategias utilizadas por la corteza para extraer información acerca de la orientación de los estímulos a partir de una entrada sensorial, la procedente del tálamo, que carece de ese atributo. Las nuevas técnicas empleadas, principalmente experimentos in vivo y computación neuronal, están produciendo un aluvión de información, a pesar de lo cual sigue sin existir un consenso sobre cuáles son los mecanismos básicos, reflejado en la existencia de diversas teorías y multitud de modelos [3-8]. En este trabajo presentamos una síntesis de los conocimientos y teorías actuales acerca de la función de V1, junto con recientes descubrimientos que han sido posibles gracias a la utilización de nuevas técnicas de imagen en combinación con registros electrofisiológicos.

\section{LA SELECTIVIDAD A LA ORIENTACIÓN COMO MODELO DE COMPUTACIÓN CORTICAL}

La electrofisiología ha demostrado que la mayoría de neuronas de V1 de al menos carnívoros y primates son extraordinariamente sensibles a la presentación en el campo visual de líneas con una determinada orientación, de tal manera que cada célula cortical produce una respuesta de salida (potenciales de acción) máxima al ser estimulada con un estrecho rango de orientaciones, permaneciendo silente o generando una respuesta mucho menor ante el resto de orientaciones. Por el contrario, las neuronas de proyección del núcleo geniculado lateral dorsal del tálamo (NGLd), que envían a la corteza la información sensorial procedente de la retina, no poseen esta propiedad; se trata por lo tanto de una función que se genera en las células corticales. A pesar de que, en principio, el estudio de la selectividad a la orientación parece fácilmente abordable desde el punto de vista experimental -a diferencia de otros procesos corticales implicados en funciones cognitivas de mayor rango-, está resultando más difícil de lo que se podría pensar 40 años atrás cuando se descubrieron las propiedades básicas de las células de V1. ¿Cómo se genera en V1 la selectividad a la orientación? ¿Cuál es la circuitería tálamo-cortical y córtico-cortical que da lugar a dicha propiedad? ¿Es una propiedad que surge directamente de unas conexiones sinápticas específicas y, en cierto modo, 
preestablecidas o es el producto emergente del funcionamiento de la corteza visual primaria como un todo? ¿Qué mecanismos permiten integrar en el mismo espacio cortical esta propiedad junto con las otras características básicas de V1? Aunque todavía no hay respuestas definitivas a estas preguntas, los experimentos y teorías que se describen a continuación pretenden ofrecer una visión general de los avances que se han realizado en los últimos años acerca de la generación de selectividad a la orientación.

El animal del que mejor se conoce la fisiología de su sistema visual es el gato y actualmente sigue siendo el modelo elegido para realizar la mayoría de los estudios acerca de la función cortical básica (estudios en los que no es necesario invocar funciones superiores asociadas a la toma de decisiones, memoria, etc, experimentos que se llevan a cabo generalmente en monos despiertos y previamente entrenados). La mayoría de los resultados comentados en el presente trabajo hacen referencia a la corteza visual del gato.

\section{DESENMASCARANDO LA FUNCIÓN CORTICAL: TÉCNICAS, TEORÍAS Y MODELOS PARA EXPLICAR UNA PROPIEDAD 'SIMPLE'}

La selectividad a la orientación fue descrita por Hubel y Wiesel [2] en experimentos en los que se estudió la corteza visual de gatos mediante el registro de la actividad extracelular con electrodos de tungsteno. Esta técnica ha sido utilizada con éxito hasta la actualidad en experimentos dirigidos a revelar los patrones temporales de descarga de potenciales de acción y la relación entre la actividad de las neuronas corticales y talámicas [9-11]. Los estímulos visuales utilizados en la mayoría de estudios sobre la selectividad a la orientación son barras individuales o grupos de barras claras y oscuras (enrejados), de distintas orientaciones, que se desplazan por delante del campo visual con una dirección ortogonal a la orientación (el recuadro en la figura 3 muestra un esquema de un gato y un enrejado de barras verticales que se desplaza hacia la derecha de su campo visual). Se puede relacionar cada estímulo presentado con la actividad neuronal registrada y construir lo que se denomina una curva de orientación. La figura 1 muestra una curva típica de una célula de V1: las distintas orientaciones (en el eje de abcisas) se representan en función del número medio de potenciales de acción que esa célula produce para cada una de ellas (ordenadas). En este caso la célula responde de manera preferente a barras verticales. Además, el mismo protocolo revela otra propiedad característica de las neuronas de la corteza visual, la selectividad a la dirección: en el ejemplo de la figura 1 la respuesta es de mayor amplitud al presentar barras verticales que se mueven hacia la derecha del campo visual $\left(90^{\circ}\right)$ que al estimular mediante barras verticales que se desplazan en la dirección contraria $\left(270^{\circ}\right)$.

Los registros extracelulares proporcionan información acerca de la frecuencia de descarga de potenciales de acción, pero no indican qué integración subumbral de las entradas sinápticas es la que origina dicha salida. Recientemente se ha conseguido aplicar con éxito in vivo la técnica de registro intracelular de célula entera [12,13], inicialmente desarrollada para preparaciones in vitro. Los registros de las variaciones del potencial de membrana (Vm) así obtenidos revelan la exuberante actividad subumbral que continuamente están produciendo las neuronas de V1, actividad que eventualmente será convertida en potenciales de acción en virtud de la integración somática. La figura 2 es un ejemplo del registro intracelular de célula entera en gato in vivo de una neurona de V1, durante la estimulación visual con enrejados móviles. Al igual que ocurre con la neurona de la figura 1, la célula responde preferentemente a barras verticales (tra-

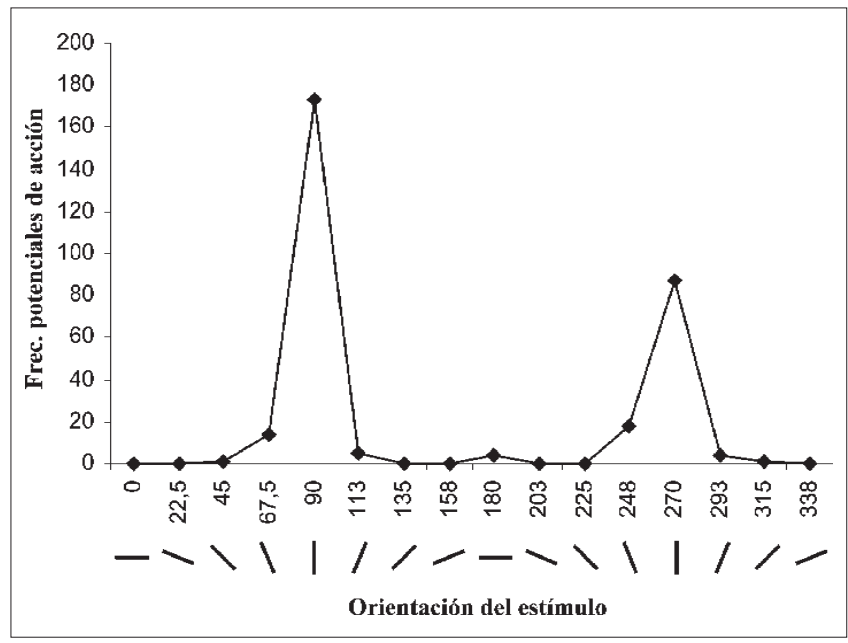

Figura 1. Curva de orientación de una célula de V1. Se representa la frecuencia media de potenciales de acción que la neurona genera ante la estimulación con enrejados de diferentes orientaciones. El análisis indica que la célula es selectiva para estímulos verticales.

zo 1), generando varios potenciales de acción. Por el contrario, la presentación del estímulo ortogonal al preferido no da lugar a ningún potencial de acción (trazo 3). Además, estos registros intracelulares permiten observar supuestos potenciales excitadores postsinápticos (PEPS) inducidos por el estímulo óptimo, así como la disminución de la amplitud de la despolarización al variar ligeramente la orientación preferida (trazo 2). En este ejemplo, la presentación del estímulo ortogonal (trazo 3) no produce despolarizaciones del $\mathrm{Vm}$, pero sí pequeñas hiperpolarizaciones (flechas).

Actualmente no hay consenso sobre cuáles son los mecanismos de integración de V1. Se han propuesto tres modelos básicos para explicar la selectividad a la orientación:

1. Modelos jerárquicos de alimentación hacia delante. Proponen que la selectividad de una célula simple de capa 4 surge directamente como consecuencia de la disposición en línea de los campos receptores de las células talámicas que envían sus conexiones excitadoras a ésta [2].

2. Modelos de inhibición por orientación cruzada. Proponen que la entrada talámica neta a una neurona cortical carece de selectividad y que las respuestas a las orientaciones no óptimas son canceladas por las entradas inhibidoras procedentes de otras células corticales con orientaciones preferentes ortogonales [14-16].

3. Modelos recurrentes. Proponen que existe un cierto sesgo de las entradas talámicas a una neurona cortical hacia una determinada orientación, el cual es amplificado por entradas locales excitadoras procedentes de otras neuronas de la misma columna cortical que comparten la misma preferencia a la orientación [17-19].

Estos dos últimos modelos se denominan también en conjunto 'modelos corticales', ya que confieren mayor importancia a las influencias procedentes de la propia corteza que a las talámicas. Cada uno de estos modelos ha recibido cierto apoyo experimental, lo que ha dado lugar a un interesante debate para definir con precisión cuáles son realmente los mecanismos de selectividad a la orientación, si es que en realidad se pueden ajustar a un único esquema, lo que parece improbable.

Respecto a los modelos de alimentación hacia delante, hay bastantes evidencias experimentales de que las entradas talámicas a una 


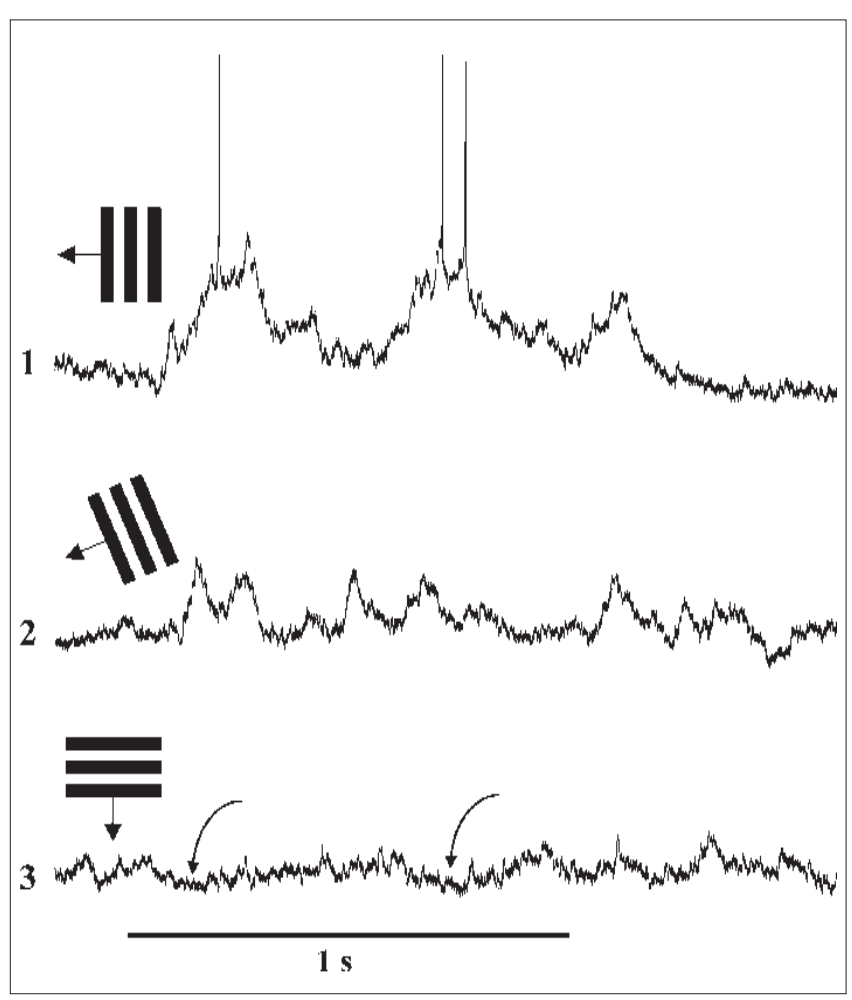

Figura 2. Registros intracelulares de célula entera de una neurona de V1. Los enrejados representados a la izquierda indican la orientación y dirección del estímulo para cada trazo. La barra inferior indica la duración de la estimulación visual. Más detalles en el texto.

célula de V1 están, de hecho, alineadas de manera específica a lo largo del eje de su orientación preferida y que ello es suficiente para conferir cierta selectividad a la orientación [10,20,21]. La utilización de registros intracelulares está resultando muy útil para conocer los efectos sinápticos de las neuronas talámicas sobre las células de capa 4, así como las interacciones de estas últimas con los siguientes eslabones del procesamiento cortical [22]. Los resultados apoyan esta idea de procesamiento jerárquico, en donde las neuronas talámicas contactan y modulan la actividad de células simples corticales, que a su vez envían conexiones a las células complejas [23,24]. Sin embargo estas entradas 'hacia delante' no pueden explicar diversas propiedades no lineales de las células de V1, así como tampoco explican de manera eficaz una particularidad de la selectividad a la orientación, la persistencia de dicha selectividad cuando se varía el contraste del estímulo -aunque recientemete se han presentado variaciones del modelo que tratan de solucionar el problema [8,25]-. También hay nuevos datos experimentales que explican en parte cómo se origina la selectividad a la orientación de las neuronas de V1 de segundo orden a partir de las entradas que reciben [26], aunque todavía es preciso integrar este esquema con la enorme cantidad de entradas sinápticas procedentes de otras regiones corticales.

Por su parte, los modelos corticales basan gran parte de sus argumentos en la enorme diversidad de tipos celulares e interconexiones que existen en la corteza. Para comprender la función de una neurona, aparte de los efectos inducidos directamente por el tálamo, es preciso considerar las conexiones procedentes de otras regiones corticales, ya que éstas suponen de manera abrumadora la principal entrada sináptica: el $95 \%$ de las sinapsis que reciben las células de capa 4 del gato provienen de otras neuronas corticales; por su parte, las células de capa 3 reciben un $75 \%$ de conexiones de otras neuronas de la misma capa [5]. Por lo tanto, los modelos de computación cortical deben de incluir y explicar el papel de estas conexiones laterales. Las células de V1 reciben dos tipos básicos de contactos laterales, por una parte conexiones corticales horizontales de larga distancia, procedentes principalmente de regiones con preferencias a la orientación similares pero con campos receptores que no se solapan [27-29]. El otro tipo son las conexiones locales, que parecen ser mucho menos específicas y más estereotipadas, resultando por lo tanto independientes a la orientación [29-32]. Según esto, debido a la gran variedad de conexiones que confluyen sobre una misma célula cortical, es fundamental conocer la naturaleza de los efectos sinápticos que las distintas entradas (talámicas, corticales locales y de larga distancia) producen sobre estas neuronas y su papel en la respuesta final (potenciales de acción) de la célula.

Probablemente la mayor disputa que existe en estos momentos está en asignar el papel que la excitación y la inhibición juega en la integración de las neuronas de V1. Por un lado, varios grupos han presentado evidencias de que tanto excitación como inhibición alcanzan la máxima amplitud en respuesta a la orientación preferida [33-36]. Pero también hay resultados experimentales que indican una mayor variabilidad en el papel de los efectos sinápticos, incluyendo neuronas en las que la inhibición es máxima para orientaciones distintas a la óptima [37-40]. Una sencilla explicación a tal diversidad sería que, debido a la heterogeneidad estructural, las neuronas de V1 no generan todas la selectividad a la orientación mediante lo mismos mecanismos [5,6,41]. ¿Sencilla? A pesar del esfuerzo que se está llevando a cabo para conocer la arquitectura fina de las distintas capas corticales y el papel de los distintos tipos funcionales básicos de células (simples, complejas, etc), parece que tampoco está siendo fácil para los investigadores de V1 ponerse de acuerdo respecto al grado de diversidad de la microcircuitería cortical $[1,42]$.

\section{PROFUNDIZANDO EN LA ESTRUCTURA FUNCIONAL DE V1 MEDIANTE NUEVAS TÉCNICAS DE IMAGEN}

Para dar respuesta a todos estos interrogantes es importante combinar la investigación acerca de la actividad neuronal individual y de los microcircuitos en los que ésta se desarrolla con el conocimiento de la organización funcional global de la corteza cerebral. En los mamíferos, las neuronas que comparten una determinada función suelen estar agrupadas; en el caso de V1 las células que responden a una determinada orientación forman columnas perpendiculares a la superficie cortical, de tal manera que si se avanza con un electrodo de registro a través de las distintas capas corticales siguiendo una de estas columnas, todas las células presentarán la misma orientación preferente, y lo mismo ocurre para otras propiedades corticales básicas. La organización columnar de la corteza fue demostrada por Mountcastle [43] y estudiada en V1 por Hubel y Wiesel [44]. Hasta hace muy poco se ha venido utilizando como referencia el modelo cúbico propuesto por éstos últimos, pero las técnicas de imagen óptica han revelado una organización distinta.

Se han desarrollado varias técnicas que permiten visualizar la actividad de grandes grupos de neuronas, como la tomografía por emisión de positrones (PET) o la imagen por resonancia magnética (fMRI). Aunque se están aplicando con éxito, permitiendo la localización tridimensional de regiones activas del cerebro, de momento ofrecen una resolución temporal y espacial baja. Estas deficiencias se están subsanando, al menos en la investigación experimental, mediante la incorporación de dos nuevas técnicas de imagen óptica: 


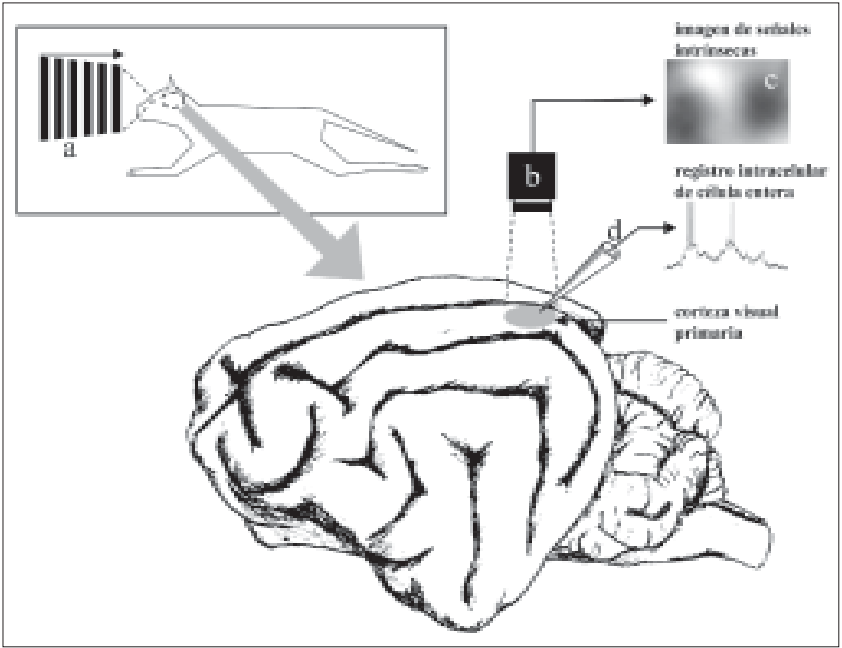

Figura 3. Esquema del diseño experimental, que combina la técnica de imagen óptica de señales intrínsecas con registros intracelulares. El animal de experimentación se sitúa enfrente de un monitor en el que se presenta el estímulo (a). Se adquieren dos tipos de señal: una imagen óptica de la actividad eléctrica de V1 evocada por el estímulo (c) a través de una cámara digital (b), y un registro de la actividad intracelular de neuronas de $\mathrm{V} 1$ a través de microelectrodos de vidrio (d).

1. Imagen basada en señales intrínsecas, que ofrece la mayor resolución espacial que se ha conseguido hasta el momento in vivo.

2. Imagen basada en colorantes sensibles al voltaje, la cual permite registrar la actividad cortical con excelentes resoluciones temporal y espacial.

La imagen óptica de señales intrínsecas se basa en la medición de los pequeños cambios en las propiedades ópticas del tejido cerebral que tienen lugar cuando éste se activa, variaciones que permiten estudiar la función cortical ya que existe una relación directa entre la actividad neuronal, la actividad metabólica local y el flujo sanguíneo [45-49]. El procedimiento para la obtención de estas imágenes es sorprendentemente simple [50]: la región de la corteza cerebral a estudiar se ilumina con luz de una determinada longitud de onda a la vez que una cámara obtiene fotografías de esa región mientras se realiza estimulación sensorial. El objetivo es detectar los cambios en la intensidad de luz reflejada debidos a la actividad neuronal; el problema es que estos cambios son minúsculos, ya que constituyen entre un 0,1 y un $6 \%$ de la intensidad total de luz reflejada. Así que, para poner a punto la técnica, a pesar de la sencillez de la teoría, hubo que esperar a que la tecnología permitiera la fabricación de cámaras de video digitales de gran velocidad y alta resolución, junto con ordenadores lo suficientemente potentes para procesar las imágenes. Las señales intrínsecas que se pueden medir son de distinta naturaleza, dependiendo de la longitud de onda con la que se ilumina la corteza. Tras la estimulación sensorial se producen varios cambios que son susceptibles de ser utilizados para la obtención de imágenes de la actividad neuronal: existe un incremento inicial de la concentración de deoxihemoglobina, resultado del aumento de consumo de oxígeno por las neuronas que han incrementado su actividad metabólica; a continuación se produce un incremento en el flujo sanguíneo que origina una disminución de la concentración de deoxihemoglobina, pues la sangre que llega al tejido que está siendo activado contiene niveles altos de oxihemoglobina; un tercer componente se debe a los cambios en el volumen sanguíneo, debido al aumento del flujo capilar local y a la dilatación

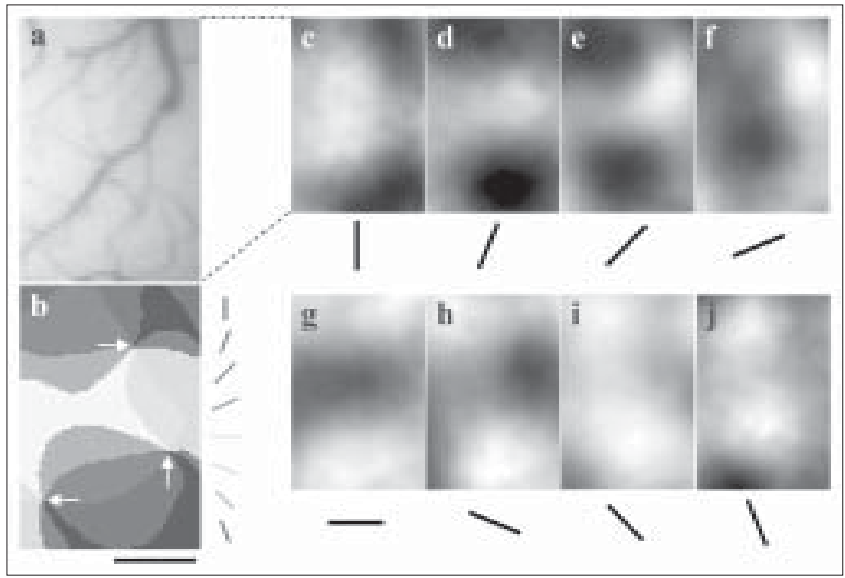

Figura 4. Construcción del mapa de orientaciones de una región de V1. a) Imagen de la superficie cortical estudiada; b) mapa de orientaciones de la región indicada en a), en el que las flechas señalan centros de orientación c-j) imágenes de la activación cortical (en oscuro) ante la presentación de estímulos con ocho orientaciones distintas (indicadas por las barras debajo de cada imagen). Barra de calibración: $700 \mu \mathrm{m}$. Más detalles en el texto.

de vénulas; finalmente, se puede medir también la señal producida por cambios en la dispersión de luz asociados a la activación cortical, debidos al movimientos de agua e iones, variación en el volumen del espacio extracelular, expansión de los capilares y liberación de neurotransmisores.

\section{EL MAPA DE ORIENTACIONES DE V1: UN POCO DE ORDEN A VISTA DE PÁJARO}

Nuestro grupo de investigación está utilizando esta técnica, combinada con registros intracelulares, para tratar de desenmascarar los mecanismos de selectividad a la orientación de las células de V1. La figura 3 muestra de forma esquemática el preparado experimental: el registro se realiza en gatos anestesiados con isofluorano en cuyo campo visual se presentan enrejados móviles con distintas orientaciones (a). Simultáneamente, una cámara digital de alta resolución (b) toma imágenes de una pequeña región de la corteza visual iluminada con luz de entre 605-630 nm de longitud de onda y enfocada entre 500-600 $\mu \mathrm{m}$ de profundidad. Posteriormente, y en función del mapa cortical obtenido (c), se introducen micropipetas de vidrio (d) en zonas concretas de la corteza para estudiar la actividad sináptica de neuronas individuales.

La utilización de señales intrínsecas para estudiar la arquitectura funcional de V1 ha revelado una organización de las columnas de orientación diferente del modelo cúbico inicial de Hubel y Wiesel [51,52]. Gracias a esta técnica, la representación de la orientación de los estímulos en el córtex se conoce mejor que cualquier otra propiedad visual (exceptuando la retinotopía). El mapa bidimensional de V1 que se obtiene tras la estimulación con enrejados móviles (Fig. 4b) indica que las células que responden a una misma orientación preferente se encuentran agrupadas, pero no en columnas rectangulares. El devenir evolutivo ha deparado un resultado más 'artístico' para organizar un número finito de células con un amplio abanico de funciones en una reducida región de la corteza cerebral: las células con una misma orientación preferente se agrupan en los denominados dominios de iso-orientación, que se organizan en círculos alrededor de singularidades denominadas centros de orientación o molinillos. De esta manera, sobre un centro de orientaciones confluyen todos los dominios de iso-orientación 


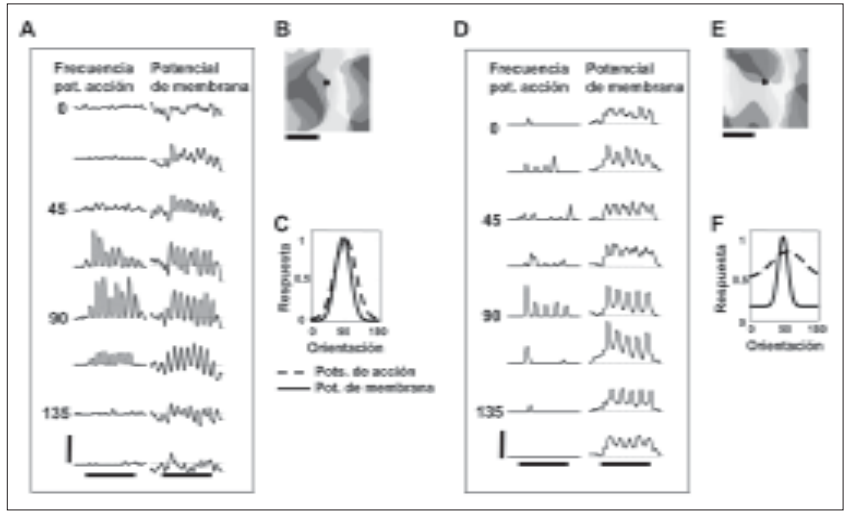

Figura 5. Frecuencia media de potenciales de acción ( $A$ y $D$, columnas de la izquierda) y actividad media del Vm (A y D, columnas de la derecha) registradas en dos células de $V 1$. La célula de la izquierda se registró en un dominio de isoorientación (B) y presenta curvas de orientación similares para la respuesta de potenciales de acción y del Vm (C). Por el contrario, la célula de la derecha se registró en un centro de orientación $(E)$; la curva de orientación para el Vm ( $F$, línea discontinua) indica una menor selectividad a la orientación que la curva para la respuesta de potenciales de acción ( $F$, línea continua). Barras verticales en A: 8 espigas/s y $10 \mathrm{mV}$. Barras verticales en D: 3 espigas/s y $8 \mathrm{mV}$. Barras horizontales en $A$ y D: 2 s. Barras de calibración en B y E: $500 \mu \mathrm{m}$. Más detalles en el texto. Modificada de [58], con permiso de Elsevier.

vecinos. La figura $4 \mathrm{~b}$ muestra el mapa de orientaciones de una pequeña región de V1 de gato, en donde los tonos de gris indican dominios de iso-orientación que confluyen en 3 centros de orientación (señalados con flechas). El mapa se ha obtenido de la siguiente manera: una vez realizada una craneotomía para dejar al descubierto V1, se retiró la duramadre y se estabilizó el tejido expuesto para evitar cualquier movimiento inducido por la respiración o el latido cardíaco (drenaje de líquido cefalorraquídeo a través de una cánula insertada en la cisterna magna, recubrimiento de la corteza expuesta con agarosa, etc). Sobre la zona a estudiar (Fig. 4a) se enfocó una cámara de video digital conectada a un amplificador diferencial (Imager 3001, Optical Imaging Inc.). A continuación se iluminó la corteza con una luz de intensidad estable y de $605 \mathrm{~nm}$, y se procedió a la estimulación visual con enrejados de 8 orientaciones distintas simultáneamente a la adquisión de imágenes. La sincronización de los estímulos con las imágenes obtenidas permitió obtener 8 mapas de activación, uno por cada orientación. Las imágenes c-j de la figura 4 representan la actividad (zonas oscuras) registrada durante la presentación de los enrejados con las 8 orientaciones distintas (indicadas por barras debajo de cada imagen), obtenidas tras promediar varios cientos de imágenes individuales y sustraer el ruido de fondo. Al representar la actividad evocada por las 8 orientaciones en una única imagen (superposición de los 8 mapas), asignando a la actividad de cada orientación un color diferente (distintos tonos de gris) se obtiene el mapa de orientaciones de la figura $4 \mathrm{~b}$.

\section{COMBINACIÓN DEL MAPA DE ORIENTACIONES CON LA ACTIVIDAD SINÁPTICA INDIVIDUAL}

El descubrimiento de la estructura del mapa de orientaciones de V1 está aportando pistas fundamentales para comprender los mecanismos que originan la selectividad a la orientación. Los registros electrofisiológicos de la actividad individual de neuronas de V1, tanto extracelulares como intracelulares, aportarán mucha más información si se conoce la localización de esas neuronas en el mapa de orientaciones. Así mismo, los nuevos modelos computa- cionales deben incluir dicha estructura [53]. Se están realizando estudios que tratan de buscar en las conexiones horizontales córtico-corticales el principal sustrato anatómico para el mapa funcional. Como ya se comentó, se ha observado que las proyecciones axonales de largo alcance conectan principalmente entre sí dominios de células con la misma orientación preferente, además estas proyecciones y el mapa de orientaciones presentan un desarrollo temporal similar [54]. Por lo tanto, las neuronas situadas en regiones próximas a los centros de orientación recibirán orientaciones de largo alcance procedentes de dominios con un amplio rango de orientaciones. Por el contrario, las proyecciones locales córtico-corticales son isotrópicas y no parecen estar relacionadas con la estructura del mapa de orientaciones. En consecuencia, una neurona localizada en el centro de un dominio de orientaciones recibirá conexiones locales básicamente de neuronas con la misma orientación preferente, sin embargo las células situadas en los centros de orientación recibirán conexiones locales procedentes de neuronas con distintas preferencias a la orientación, o sea, el tipo de entradas locales y de largo alcance que una neurona de V1 recibe probablemente dependa de su situación en el mapa. Según este argumento, las neuronas situadas en los centros de orientación, ¿carecen de selectividad a la orientación dado que reciben entradas sinápticas de todo el rango de orientaciones? De manera sorprendente, al realizar registros extracelulares unitarios de neuronas localizadas en dominios y en centros de orientación, se comprobó que ambos tipos de células presentan curvas de orientación similares [55]. Por lo tanto, dada la heterogeneidad estructural subyacente, es probable que ambos tipos de neuronas generen su selectividad a la orientación mediante mecanismos diferentes. Además, se ha demostrado que, dependiendo de la capa cortical en la que una neurona se encuentra, existen distintas combinaciones de efectos sinápticos despolarizantes e hiperpolarizantes que pueden dar lugar a la selectividad a la orientación [56].

En nuestro laboratorio se ha comprobado recientemente que las neuronas situadas en los centros de orientación son susceptibles de mayor plasticidad que las neuronas de los dominios y que, mediante adaptación, se puede modificar su orientación preferente [57], lo cual puede ser debido a la mayor diversidad de entradas sinápticas que reciben. Para estudiar los mecanismos sinápticos que dan lugar a la selectividad a la orientación dentro de este nuevo esquema estructural, hemos realizado experimentos de imagen óptica de señales intrínsecas combinados con el registro de la actividad subumbral de neuronas situadas en ambas regiones del mapa de orientaciones [58]. Los resultados indican que aunque efectivamente las curvas de orientación para la actividad de potenciales de acción son similares en cualquier parte del mapa de orientaciones, la actividad sináptica no lo es y muestra diferencias relacionadas con la situación de las distintas neuronas. En el ejemplo de la figura 5 se comparan los resultados obtenidos de una célula registrada en un dominio de iso-orientación (localización señalada con un punto en el mapa B) respecto a una célula registrada en un centro de orientación (punto en el mapa E). La frecuencia de descarga de potenciales de acción en ambas células (A y D, columnas de la izquierda) da lugar, como es de esperar, a curvas de orientación similares (líneas continuas en C y F). Sin embargo la actividad sináptica es distinta en ambos tipos de células: la célula situada en el dominio de iso-orientación presenta una relación lineal entre la actividad sináptica (A, columna de la derecha) y la frecuencia de potenciales de acción, de modo que las curvas de orientación para ambos parámetros son similares (C). Por el contrario, la neurona situada en el centro de orientación genera ante la 
estimulación visual una respuesta sináptica más compleja, caracterizada por la existencia de despolarización del Vm tanto para la estimulación con la orientación preferida como para el resto de orientaciones, lo que origina una curva de orientaciones menos aguda ( $\mathrm{F}$, línea discontinua); sin embargo, solamente un estrecho rango de orientaciones despolariza la célula lo suficiente como para generar potenciales de acción, por lo que la curva de orientaciones para la frecuencia de espigas mantiene el mismo perfil que en los dominios. Estos resultados sugieren que las neuronas situadas en los centros de orientación deben de poseer mecanismos de ajuste para filtrar con eficacia el amplio rango de entradas sinápticas que reciben, manteniendo así su agudeza en la discriminación de la orientación del estímulo. En estos experimentos también se demostró que la selectividad a la orientación del potencial de membrana está directamente relacionada con las entradas sinápticas locales, y que es posible calcular de manera teórica dicha selectividad en función del mapa de orientaciones.

El protocolo de registros intracelulares utilizado en estos experimentos no permite obtener información acerca del tipo concreto y la intensidad de los efectos sinápticos (excitación/inhibición) que se producen en cada momento. Esto es así porque el registro pasivo del Vm indica la existencia de despolarizaciones o inhibiciones, pero no permite identificar el tipo de fuerzas sinápticas que originan dichos cambios (ya que, por ejemplo, una despolarización puede deberse a la existencia de excitación, al cese de una inhibición o a una mezcla de ambos efectos). Para revelar la naturaleza de los efectos sinápticos que tienen lugar en una determinada neurona es necesario modificar artificialmente el $\mathrm{Vm}$ de la célula mediante la inyección de corriente, de modo que éste se acerque o se aleje de los potenciales de equilibrio para los distintos iones implicados. Esta estrategia permite separar de manera precisa las conductancias inhibidoras de las excitadoras, lo cual, como ya se comentó anteriormente, es uno de los principales objetivos actuales en el estudio de V1. Recientemente se han publicado varios trabajos en donde se han medido estos cambios de conductancia asociados a la selectividad a la orientación [36,40], pero los resultados son dispares, en parte porque los registros realizados no se han relacionado con la arquitectura cortical.

En síntesis, los resultados más recientes apuntan a la existencia de distintos mecanismos capaces de generar la selectividad a la orientación de las células de V1, los cuales dependen de la arquitectura del mapa de orientaciones o de la situación de las neuronas en las distintas capas corticales. Por lo tanto, para definir con más precisión los mecanismos que generan dicha selectividad, uno de los pasos siguientes debe de consistir en relacionar las mediciones de cambios de conductancia con los distintos tipos de heterogeneidades estructurales.

\section{CONCLUSIONES}

El aumento de la resolución espacial y temporal de las técnicas de muestreo han incrementado en varios grados de magnitud la información acerca de la estructura y función de la corteza cerebral, generando nuevas teorías y modelos que tratan de dar respuesta a la extrema complejidad observada.

El estudio de la selectividad a la orientación de las células de V1 es uno de los principales modelos que la neurociencia actual está utilizando para comprender los mecanismos de integración de información realizados por la corteza cerebral. A pesar de que, desde el punto de vista funcional, es una propiedad básica y fácil de registrar, la arquitectura y computación subyacentes están resultando difíciles de descifrar. El estudio actual de esta y otras propiedades corticales se está llevando a cabo mediante un refinamiento de los registros tradicionales extracelulares e intracelulares y su combinación con nuevas técnicas anatómicas y de imagen que permiten obtener una visión global y a la vez precisa de la arquitectura cortical. El registro de los efectos sinápticos asociados a la selectividad a la orientación en combinación con la anatomía y arquitectura cortical indican que probablemente exista más de un mecanismo capaz de generar dicha propiedad.

\section{BIBLIOGRAFÍA}

1. Silberberg G, Gupta A, Markram H. Stereotypy in neocortical microcircuits. Trends Neurosci 2002; 25: 227-30.

2. Hubel DH, Wiesel TN. Receptive fields, binocular interaction and functional architecture in the cat's visual cortex. J Physiol 1962; 160: 106-54.

3. Sompolinsky H, Shapley R. New perspectives on the mechanisms for orientation selectivity. Curr Opin Neurobiol 1997; 7: 514-22.

4. Ferster D, Miller KD. Neural mechanisms of orientation selectivity in the visual cortex. Annu Rev Neurosci 2000; 23: 441-71.

5. Martin KA. Microcircuits in visual cortex. Curr Opin Neurobiol 2002; 12: 418-25

6. Vidyasagar TR, Pei X, Volgushev M. Múltiple mechanisms underlying the orientation selectivity of visual cortical neurons. Trends Neurosci 1996; 19: 272-7.

7. Reid RC, Alonso JM. The processing and encoding of information in the visual cortex. Curr Opin Neurobiol 1996; 6: 475-80.

8. Miller KD. Understanding layer 4 of the cortical circuit: a model based on cat V1. Cereb Cortex 2003; 13: 73-82.

9. Tanaka K. Cross-correlation analysis of geniculostriate neuronal relationships in cats. J Neurophysiol 1983; 49: 1303-18.

10. Reid RC, Alonso JM. Specificity of monosynaptic connections from thalamus to visual cortex. Nature $1995 ; 378: 281-4$.

11. Alonso JM, Usrey WM, Reid WC. Precisely correlated firing in cells of the lateral geniculate nucleus. Nature 1996; 383: 815-9.

12. Pei X, Volgushev M, Vidyasagar TR, Creutzfeldt OD. Whole cell recording and conductance measurements in cat visual cortex in-vivo. Neuroreport 1991; 2: 485-8.

13. Margrie TW, Brecht M, Sakmann B. In vivo, low-resistance, whole-cell recordings from neurons in the anaesthetized and awake mammalian brain. Pflugers Arch 2002; 444: 491-8.

14. Sillito AM. The contribution of inhibitory mechanisms to the receptive field properties of neurons in the striate cortex of the cat. J Physiol 1975; 250: 305-29.

15. Morrone MC, Burr DC, Maffei L. Functional implications of crossorientation inhibition of cortical visual cells. I. Neurophysiological evidence. Proc R Soc Lond B Biol Sci 1982; 216: 335-54.

16. Bonds AB. Role of inhibition in the specification of orientation selectivity of cells in the cat striate cortex. Vis Neurosci 1989; 2: 41-55.

17. Ben-Yishai R, Bar-Or RL, Sompolinsky H. Theory of orientation tuning in visual cortex. Proc Natl Acad Sci USA 1995; 92: 3844-8.

18. Douglas RJ, Koch C, Mahowald M, Martin KA, Suárez HH. Recurrent excitation in neocortical circuits. Science 1995; 269: 981-5.

19. Somers DC, Nelson SB, Sur M. An emergent model of orientation selectivity in cat visual cortical simple cells. J Neurosci 1995; 15: 5448-65.

20. Chapman B, Zahs KR, Stryker MP. Relation of cortical cell orientation selectivity to alignment of receptive fields of the geniculocortical afferents that arborize within a single orientation column in ferret visual cortex. J Neurosci 1991; 11: 1347-58.

21. Troyer TW, Krukowski AE, Priebe NJ, Miller KD. Contrast-invariant orientation tuning in cat visual cortex: thalamocortical input tuning and correlation-based intracortical connectivity. J Neurosci 1998; 18: 5908-27.

22. Hirsch JA. Synaptic physiology and receptive field structure in the early visual pathway of the cat. Cereb Cortex 2003; 13: 63-9.

23. Hirsch JA, Alonso JM, Reid RC, Martínez LM. Synaptic integration in striate cortical simple cells. J Neurosci 1998; 18: 9517-28.

24. Hirsch JA, Martínez LM, Alonso JM, Desai K, Pillai C, Pierre C. Synaptic physiology of the flow of information in the cat's visual cortex in vivo. J Physiol 2002; 540: 335-50.

25. Anderson JS, Lampl I, Gillespie DC, Ferster D. The contribution of noise to contrast invariance of orientation tuning in cat visual cortex. Science 2000; 290: 1968-72. 
26. Alonso JM, Martínez LM. Functional connectivity between simple cells and complex cells in cat striate cortex. Nat Neurosci 1998; 1: 395-403.

27. Ts'o DY, Gilbert CD, Wiesel TN. Relationships between horizontal interactions and functional architecture in cat striate cortex as revealed by cross-correlation analysis. J Neurosci 1986; 6: 1160-70.

28. Gilbert CD, Wiesel TN. Columnar specificity of intrinsic horizontal and corticocortical connections in cat visual cortex. J Neurosci 1989; 9: 2432-42.

29. Bosking WH, Zhang Y, Schofield B, Fitzpatrick D. Orientation selectivity and the arrangement of horizontal connections in tree shrew striate cortex. J Neurosci 1997; 17: 2112-27.

30. Malach R, Amir Y, Harel M, Grinvald A. Relationship between intrinsic connections and functional architecture revealed by optical imaging and in vivo targeted biocytin injections in primate striate cortex. Proc Natl Acad Sci USA 1993; 90: 10469-73.

31. Kisvarday ZF, Toth E, Rausch M, Eysel UT. Orientation-specific relationship between populations of excitatory and inhibitory lateral connections in the visual cortex of the cat. Cereb Cortex 1997; 7: 605-18.

32. Das A, Gilbert CD. Topography of contextual modulations mediated by short-range interactions in primary visual cortex. Nature 1999; 399: 655-61.

33. Ferster D. Orientation selectivity of synaptic potentials in neurons of cat primary visual cortex. J Neurosci 1986; 6: 1284-301.

34. Ferster D. Origin of orientation-selective EPSPs in simple cells of cat visual cortex. J Neurosci 1987; 7: 1780-91.

35. Nelson S, Toth L, Sheth B, Sur M. Orientation selectivity of cortical neurons during intracellular blockade of inhibition. Science 1994; 265: 774-7.

36. Anderson JS, Carandini M, Ferster D. Orientation tuning of input conductance, excitation and inhibiton in cat primary visual cortex. J Neurophysiol 2000; 84: 909-26.

37. Volgushev M, Pei X, Vidyasagar TR, Creutzfeld OD. Excitation and inhibition in orientation selectivity of cat visual cortex neurons revealed by whole-cell recordings in vivo. Vis Neurosci 1993; 10: 1151-5.

38. Pei X, Vidyasagar TR, Volgushev M, Creutzfeldt OD. Receptive field analysis and orientation selectivity of postsynaptic potentials of simple cells in cat visual cortex. J Neurosci 1994; 14: 7130-40.

39. Borg-Graham LJ, Monier C, Fregnac Y. Visual input evokes transient and strong shunting inhibition in visual cortical neurons. Nature 1998; 393: 369-73.

40. Monier C, Chavane F, Baudot P, Graham LJ, Fregnac Y. Orientation and direction selectivity of synaptic inputs in visual cortical neurons: a diversity of combinations produces spike tuning. Neuron 2003; 37: 663-80.

\section{COMBINACIÓN DE NUEVAS TÉCNICAS \\ ELECTROFISIOLÓGICAS Y DE IMAGEN EN EL ESTUDIO DE LA FUNCIÓN DE LA CORTEZA VISUAL PRIMARIA}

Resumen. Objetivo. Se realiza una síntesis de las investigaciones que se llevan a cabo para tratar de explicar la selectividad a la orientación (SO) de la corteza visual primaria (V1), así como una descripción de las técnicas electrofisiológicas y de imagen que se utilizan. Desarrollo. El estudio de la SO de V1 es clave para comprender los mecanismos corticales de integración de la información, pero su desciframiento resulta complejo y, de momento, no hay consenso sobre cuál es el modelo que explica mejor la actividad de las neuronas de V1. Las investigaciones actuales se centran en averiguar el peso específico de las distintas entradas que puede recibir una célula cortical, tanto talámicas como procedentes de conexiones corticales laterales, y su relación con la arquitectura de la corteza. Para ello, se combinan técnicas de imagen óptica de señales intrínsecas con el registro intracelular de neuronas de V1. Experimentos recientes han demostrado diferencias entre la integración sináptica que realizan las neuronas localizadas en los dominios de isoorientación y las de los centros de orientación del mapa funcional de V1. Conclusiones. La información acerca de la actividad sináptica, combinada con la estructura funcional cortical, aporta nuevas pistas sobre la computación realizada por $V 1$, las cuales sugieren que existe más de un mecanismo capaz de generar SO. [REV NEUROL 2003; 36: 944-50] Palabras clave. Corteza visual primaria. Efectos sinápticos. Imagen óptica de señales intrínsecas. Intracelular. Mapa de orientaciones. V1.
41. Volgushev M, Pernberg J, Eysel UT. Comparison of the selectivity of postsynaptic potentials and spike responses in cat visual cortex. Eur $\mathbf{J}$ Neurosci 2000; 12: 257-63.

42. Nelson S. Cortical microcircuits: diverse or canonical? Neuron 2002 ; 36: 19-27.

43. Mountcastle VB. Modality and topographic properties of single neurons of cat's somatic sensory cortex. J Neurophysiol 1957; 20: 408-34

44. Hubel D, Wiesel T. Functional architecture of macaque monkey visual cortex. Proc R Soc Lond B Biol Sci 1977; 198: 1-59.

45. Kety SS, Landau WM, Freygang WH, Rowland LP, Sokoloff L. Estimation of regional circulation in the brain by uptake of an inert gas. American Physiological Society Abstr 1955; 1: 85.

46. Lassen NA, Ingvar DH. The blood flow of the cerebral cortex determined by radioactive krypton. Experimentia Basel 1961; 17: 42-3.

47. Sokoloff L. Relation between physiological function and energy metabolism in the central nervous system. J Neurochem 1977; 19: 13-26.

48. Raichle ME, Martin WRW, Herscovitz P, Minton MA, Markham JJ. Brain blood flow measured with intravenous H2(15)0. II. Implementation and validation. J Nucl Med 1983; 24: 790-8.

49. Fox PT, Mintun MA, Raichle ME, Miezin FM, Allman JM, van Essen DC. Mapping human visual cortex with positron emission tomography. Nature 1986; 323: 806-9.

50. Bonhoeffer T, Grinvald A. Optical imaging based on intrinsic signals: the methodology. In Toga AW, Mazziotta JC, eds. Brain mapping; the methods. San Diego: Academic Press; 1996. p. 55-97.

51. Bonhoeffer T, Grinvald A. Iso-orientation domains in cat visual cortex are arranged in pinwheel-like patterns. Nature 1991; 353: 429-31.

52. Blasdel GG. Orientation selectivity, preference and continuity in monkey striate cortex. J Neurosci 1992; 12: 3139-61.

53. Kang K, Shelley M, Sompolinsky H. Mexican hats and pinwheels in visual cortex. Proc Natl Acad Sci USA 2003; 100: 2848-53.

54. Sur M, Leamey CA. Development and plasticity of cortical areas and networks. Nat Rev Neurosci 2001; 2: 251-62.

55. Maldonado PE, Godecke I, Gray CM, Bonhoeffer T. Orientation selectivity in pinwheel centers in cat striate cortex. Science 1997; 276: 1551-5.

56. Martínez LM, Alonso JM, Reid RC, Hirsch JA. Laminar processing of stimulus orientation in cat visual cortex. J Physiol 2002; 540: 321-33.

57. Dragoi V, Rivadulla C, Sur M. Foci of orientation plasticity in visual cortex. Nature 2001; 411: 80-6.

58. Schummers J, Mariño J, Sur M. Synaptic integration by V1 neurons depends on location within the orientation map. Neuron 2002; 36: 969-78.

\section{COMBINAÇÃO DE NOVAS TÉCNICAS ELECTROFISIOLÓGICAS E DE IMAGEM NO ESTUDO DA FUNÇÃO DO CÓRTEX VISUAL PRIMÁRIO}

Resumo. Objectivo. Realiza-se uma síntese das investigações que se efectuam para tentar explicar a selectividade à orientação (SO) do córtex visual primário (V1), assim como uma descrição das técnicas electrofisiológicas e de imagem que se utilizam. Desenvolvimento. O estudo da SO de VI é a chave para compreender os mecanismos corticais de integração da informação, mas a sua decifração é complexa e, de momento, não há consenso sobre qual é o modelo que melhor explica a actividade dos neurónios de V1. As investigações actuais centram-se em conhecer o peso específico das distintas entradas que uma célula cortical pode receber, tanto talâmicas como procedentes de conexões corticais laterais, e a sua relação com a arquitectura do córtex. Para isso, combinam-se as técnicas de imagem óptica de sinais intrínsecas com o registo intracelular de neurónios de V1. Experiências recentes demonstraram diferenças na integração sináptica realizada entre neurónios localizados nos domínios da isoorientação e os centros de orientação do mapa funcional de V1. Conclusões. A informação sobre a actividade sináptica combinada com a estrutura funcional cortical contribui com novas pistas sobre o cálculo realizado por V1, as quais sugerem a existência de mais do que um mecanismo capaz de gerar SO. [REV NEUROL 2003; 36: 944-50]

Palavras chave. Córtex visual primário. Efeitos sinápticos. Imagem óptica de sinais intrínsecos. Intracelular. Mapa de orientações. V1. 